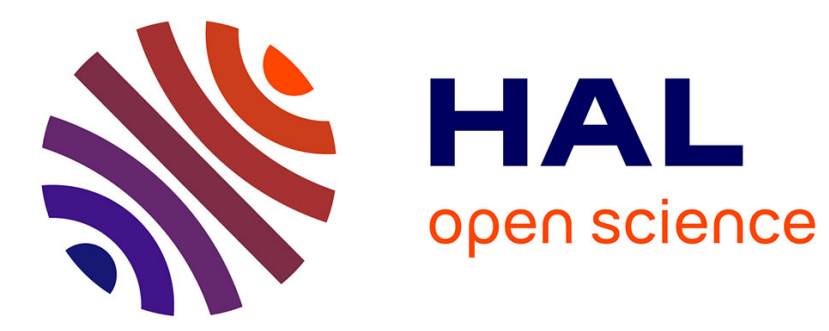

\title{
Observations of shock propagation through turbulent plasma in the solar corona
}

Eoin P Carley, Baptiste Cecconi, Hamish A Reid, Carine Briand, K Sasikumar Raja, Sophie Masson, Vladimir Dorovskyy, Caterina Tiburzi, Nicole Vilmer, Pietro Zucca, et al.

\section{To cite this version:}

Eoin P Carley, Baptiste Cecconi, Hamish A Reid, Carine Briand, K Sasikumar Raja, et al.. Observations of shock propagation through turbulent plasma in the solar corona. The Astrophysical Journal, 2021, 921, pp.3. 10.3847/1538-4357/ac1acd . hal-03367884v2

\section{HAL Id: hal-03367884 \\ https://hal.science/hal-03367884v2}

Submitted on 2 Feb 2022

HAL is a multi-disciplinary open access archive for the deposit and dissemination of scientific research documents, whether they are published or not. The documents may come from teaching and research institutions in France or abroad, or from public or private research centers.
L'archive ouverte pluridisciplinaire HAL, est destinée au dépôt et à la diffusion de documents scientifiques de niveau recherche, publiés ou non, émanant des établissements d'enseignement et de recherche français ou étrangers, des laboratoires publics ou privés.

\section{(c)(1)}

Distributed under a Creative Commons Attribution| 4.0 International License 


\title{
Observations of Shock Propagation through Turbulent Plasma in the Solar Corona
}

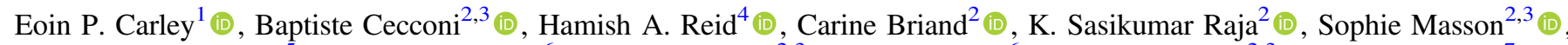 \\ Vladimir Dorovskyy ${ }^{5}$ (D), Caterina Tiburzi ${ }^{6}$ (D) , Nicole Vilmer ${ }^{2,3}$ (D), Pietro Zucca ${ }^{6}$ (D), Philippe Zarka ${ }^{2,3}$ (D), Michel Tagger ${ }^{7}$, \\ Jean-Mathias Grießmeier ${ }^{7,3}$ (D), Stéphane Corbel $^{3,8}$ (D), Gilles Theureau ${ }^{3,7,9}$ (D), Alan Loh ${ }^{2}$ (D), and Julien N. Girard ${ }^{8}$ (D) \\ ${ }^{1}$ Astronomy \& Astrophysics Section, Dublin Institute for Advanced Studies, Dublin 2, D02 XF86, Ireland; eoin.carley@dias.ie \\ LESIA, Observatoire de Paris-PSL, CNRS, Sorbonne Université, Université de Paris, Meudon, France \\ ${ }^{3}$ Station de Radioastronomie de Nançay, Observatoire de Paris, CNRS, Université d'Orléans, Nançay, France \\ ${ }^{4}$ Department of Space \& Climate Physics, University College London, UK \\ ${ }^{5}$ Institute of Radio Astronomy, National Academy of Sciences of Ukraine, Kharkov, Ukraine \\ ${ }^{6}$ ASTRON, Netherlands Institute for Radio Astronomy, Postbus 2, 7990 AA, Dwingeloo, The Netherlands \\ ${ }^{7}$ Laboratoire de Physique et Chimie de l'Environment et l'Espace, Université d'Orleans-CNRS, F-45071 Orléans cedex 2, France \\ 8 AIM, CEA, CNRS, Université de Paris, Université Paris-Saclay, F-91191 Gif-sur-Yvette, France \\ ${ }^{9}$ Laboratoire Univers et Théories, Observatoire de Paris, Université PSL, CNRS, Université de Paris, F-92190 Meudon, France \\ Received 2021 February 2; revised 2021 July 7; accepted 2021 August 3; published 2021 October 26
}

\begin{abstract}
Eruptive activity in the solar corona can often lead to the propagation of shock waves. In the radio domain the primary signature of such shocks are type II radio bursts, observed in dynamic spectra as bands of emission slowly drifting toward lower frequencies over time. These radio bursts can sometimes have an inhomogeneous and fragmented fine structure, but the cause of this fine structure is currently unclear. Here we observe a type II radio burst on 2019 March 20th using the New Extension in Nançay Upgrading LOFAR, a radio interferometer observing between $10-85 \mathrm{MHz}$. We show that the distribution of size scales of density perturbations associated with the type II fine structure follows a power law with a spectral index in the range of $\alpha=-1.7$ to -2.0 , which closely matches the value of $-5 / 3$ expected of fully developed turbulence. We determine this turbulence to be upstream of the shock, in background coronal plasma at a heliocentric distance of $\sim 2 R_{\odot}$. The observed inertial size scales of the turbulent density inhomogeneities range from $\sim 62 \mathrm{Mm}$ to $\sim 209 \mathrm{~km}$. This shows that type II fine structure and fragmentation can be due to shock propagation through an inhomogeneous and turbulent coronal plasma, and we discuss the implications of this on electron acceleration in the coronal shock.
\end{abstract}

Unified Astronomy Thesaurus concepts: Solar physics (1476); Solar coronal mass ejections (310); Solar coronal mass ejection shocks (1997); Solar particle emission (1517)

\section{Introduction}

Coronal mass ejections (CMEs) are eruptions of magnetized plasma from the solar corona into the heliosphere. These eruptions can drive shocks through the solar atmosphere, and the primary radio signature of such shocks are known as type II radio bursts (Nelson \& Melrose 1985; Mann et al. 1996). Type II bursts usually last tens of minutes and are characterized by bands of emission slowly drifting to lower frequencies over time. They can often show a fine structure, which sometimes has the appearance of fragmented, short duration, and narrow-band bursts of emission (Armatas et al. 2019). It is expected that this fragmentation is likely due to the associated shock wave propagating through inhomogeneous coronal plasma (Afanasiev 2009); however, the exact nature of the inhomogeneity has rarely been explored. A measure of the distribution of size scales of the inhomogeneity may provide insight into the turbulent nature of shocks in the corona. Type II fragmentation may therefore be important in the study of coronal turbulence, as well as the implications of turbulence on particle acceleration in the coronal shock (Guo \& Giacalone 2010).

Type II bursts are known to have a variety of different forms of substructure, which can come in the form of herringbones (Cairns \& Robinson 1987; Carley et al. 2013, 2015), as well as band

Original content from this work may be used under the terms of the Creative Commons Attribution 4.0 licence. Any further distribution of this work must maintain attribution to the author(s) and the title of the work, journal citation and DOI. splitting of either fundamental or harmonic components of the radio burst (Vršnak et al. 2001; Chrysaphi et al. 2018; Maguire et al. 2020). The bursts can also have a much less regular appearance, showing fragmentation and sporadic emission that can be broad or narrow band in frequency, particularly when they are observed with high time and frequency resolution dynamic spectra (Magdalenić et al. 2020). Given that the corona and solar wind is known to be an inhomogeneous and turbulent medium (Bale et al. 2019; Krupar et al. 2020), the sporadic fragmentation of type II bursts may due to the turbulent nature of the medium through which the associated shock propagates. Some single event and statistical studies of the small-scale structure of type II bursts have been undertaken (Magdalenić et al. 2020; Armatas et al. 2019). However to our knowledge these properties have not been studied in the context of coronal turbulence.

Theoretically, type II bursts are caused by plasma emission from beams of electrons accelerated at the shock front. The electrons are believed to be accelerated by the shock drift acceleration (SDA) mechanism (Holman \& Pesses 1983), in which the electrons gain energy while undergoing a $\nabla B$-drift in the $\vec{v} \times \vec{B}$ convective electric field of the shock (Ball \& Melrose 2001). While SDA predicts particle energy gain upon single reflection from the shock, certain hybrid models employ a combination of SDA and turbulence to guarantee multiple reflections from the shock and hence higher energy gain (Burgess 2006; Guo \& Giacalone 2010). This has been used to explain the $\sim 100 \mathrm{keV}$ energies of electrons observed at interplanetary shocks (Simnett et al. 2005), which cannot be explained by a single-reflection SDA mechanism alone. 
Turbulent plasma and inhomogeneous shocks have also been suggested as an explanation for herringbone features in type II bursts (Zlobec et al. 1993; Vandas \& Karlický 2011), which imply a time variability or quasi-periodicity to the particle acceleration mechanism. However a complete explanation of herringbone time variability still remains elusive.

Turbulence and time variability of shock properties likely play an important role in particle acceleration mechanisms in coronal shocks and the resulting appearance of type II substructure. It is only with modern radio instrumentation that we have the spectral resolution and sensitivity to probe coronal turbulence in type II fine structure as well as other burst types. For example, Chen et al. (2018) recently used the Low Frequency Array (LOFAR; van Haarlem et al. 2013) to show that type III burst fine structures have a power-law spectrum of intensity fluctuations with a spectral index of $\alpha=-1.71$. This suggests the radio burst fine structure could be related to the properties of the fully developed density turbulence through which the electron beam travels. This mechanism was partly modeled using quasi-linear theory of induced plasma emission in a turbulent coronal plasma (Reid \& Kontar 2017), showing that Langmuir wave clumping in space can be directly related to background density turbulence. Using a numerical and analytical approach combined with LOFAR observations, Reid \& Kontar (2021) recently showed that type III fine structure properties are induced by coronal density turbulence and can be used as a remote probe of this turbulence.

Here we employ the New Extension in Nançay Upgrading LOFAR (NenuFAR; Zarka et al. 2012) to study the nature of coronal turbulence in the environment of a complex type II burst during an eruptive event in the solar corona. NenuFAR has unprecedented frequency resolution of $6 \mathrm{kHz}$ in its observing range of $10-85 \mathrm{MHz}$. Given that the bandwidth of emission in frequency is related to the size scale of density structures in the corona, this allows us to study in detail the size distribution of density inhomogeneities responsible for fragmented type II emission. We show that this distribution specifically follows the signature of fully developed turbulence during shock propagating through the solar corona. In Section 2, we provide an observational overview of the type II burst, in Section 3, we show a power spectral density (PSD) analysis for three different parts of the type II burst, and finally, we discuss the nature of the observed turbulence spectrum in Section 4.

\section{Observations}

On 2019 March 20 a C4.8 class flare took place in active region AR 12736, peaking at $~ 11: 18 \mathrm{UT}$, see Figure 1. A faint EUV wave was observed during this time by the Atmospheric Imaging Assembly (AIA; Lemen et al. 2012) 171 and $193 \AA$ passbands, visible in Figure 1(a)-(c). To improve the wave visibility in the images we have used a combination of running ratio images (of 5 minute separation) and enhancement of low spatial frequency components using a Hanning window in the image Fourier domain. The wave is visible propagating both on-disk and offlimb, where it propagates with a radial sky-plane speed of $480 \pm 150 \mathrm{~km} \mathrm{~s}^{-1}$ and reaches the AIA field-of-view edge at $\sim 11: 23$ UT. Deprojecting this speed by the longitudinal angle of the source active region from the sky plane $\left(60^{\circ}\right)$, we find a speed of $950 \pm 310 \mathrm{~km} \mathrm{~s}^{-1}$. The speed uncertainty results from a $50 \mathrm{Mm}$ positional uncertainty on the EUV wave. The wave is then followed by the observation of a coronal mass ejection (CME) in the Large Angle Spectrometric Coronagraph (Brueckner et al. 1995) C2 field of view at $11: 48 \mathrm{UT},{ }^{10}$ which propagates at a constant speed of $500 \mathrm{~km} \mathrm{~s}^{-1}$ (in the sky plane).

The complex radio activity associated with this event started at $\sim 11: 05$ UT with a patchy radio emission observed at $\sim 1000 \mathrm{MHz} .{ }^{11}$ This is followed by fast-drifting type III bursts, a type II radio burst, and a broadband type IV continuum. The radio event was observed at metric to hectometric wavelengths, recorded by ground-based instruments such as the "Observations Radio pour FEDOME et l'Étude des Éruptions Solaires" (ORFEES) spectrometer and the Nançay Decametric Array (Lecacheux 2000), as well as the space-based WIND/WAVES instrument (Bougeret et al. 1995). In this study we will exclusively focus on the NenuFAR observations.

From 11:18-11:45 UT a series of complex radio bursts was observed by NenuFAR, which provided a dynamic spectrum from $20-55 \mathrm{MHz}$ during this period. ${ }^{12}$ The radio bursts in NenuFAR begins with a number of type III bursts, a broadband feature starting at 11:20 UT, followed by a complex type II burst. In our analysis below, we examine different parts of the type II burst, labeled type II part-a to part-c in the dynamic spectrum in Figure 1(e). Type II part-a starts at 11:22 UT at $\sim 45 \mathrm{MHz}$ and consists of two separate but connected series of herringbone bursts. Type II part-b is a small fragmented feature occurring at 11:22 UT and starting at $\sim 23 \mathrm{MHz}$ (we show in the next section these are a fundamental-harmonic pair). Type II part-c is a faint and fragmented structure beginning at $\sim 11: 28: 30$ UT at $\sim 30 \mathrm{MHz}$. Each part of the type II burst is morphologically different. In the following sections we analyze the size scales of density inhomogeneity in the corona that were responsible for the features of each radio burst.

\section{Methods and Results}

Our goal is to attempt to identify any evidence of turbulence being responsible for the substructure that we see in the type II burst fine structure. For this we search for a power-law distributions of the size scales associated with the inhomogeneity in the radio burst, similar to the analyses performed by Chen et al. (2018) and Reid \& Kontar (2021) for type III bursts.

Type II radio bursts are assumed to be plasma emission from mildly relativistic electrons accelerated at coronal shocks (Mann \& Klassen 2005). For plasma emission, the frequency of emission $f_{p e}$ is directly related to the electron number density $n_{e}$ in the corona $\left(f_{p e} \approx 8980 \sqrt{n_{e}}\right.$, where $n_{e}$ is in $\mathrm{cm}^{-3}$ and $f_{p e}$ is in $\mathrm{Hz}$ ). If we assume a coronal density that follows a hydrostatic equilibrium we may obtain an estimate for the altitude of the emission in the corona for any frequency using a density model. At any one time, the extent in frequency space of any spectral feature can also give the extent of the emission source in real space, provided we assume the density inhomogeneity is an enhancement of the background density model. Previous numerical modeling has also shown that density turbulence modulates the level of Langmuir waves in the plasma emission process, e.g., Reid \& Kontar (2017, 2021). Hence, we can perform a PSD analysis of a radio burst intensity variation to obtain the distribution of size scales of density perturbations.

\footnotetext{
${ }^{10}$ See https://cdaw.gsfc.nasa.gov/.

11 See http://secchirh.obspm.fr.

12 NenuFAR observation ID: 20190320_104900_20190320_125000_SUN_ TRACKING_BHR.
} 

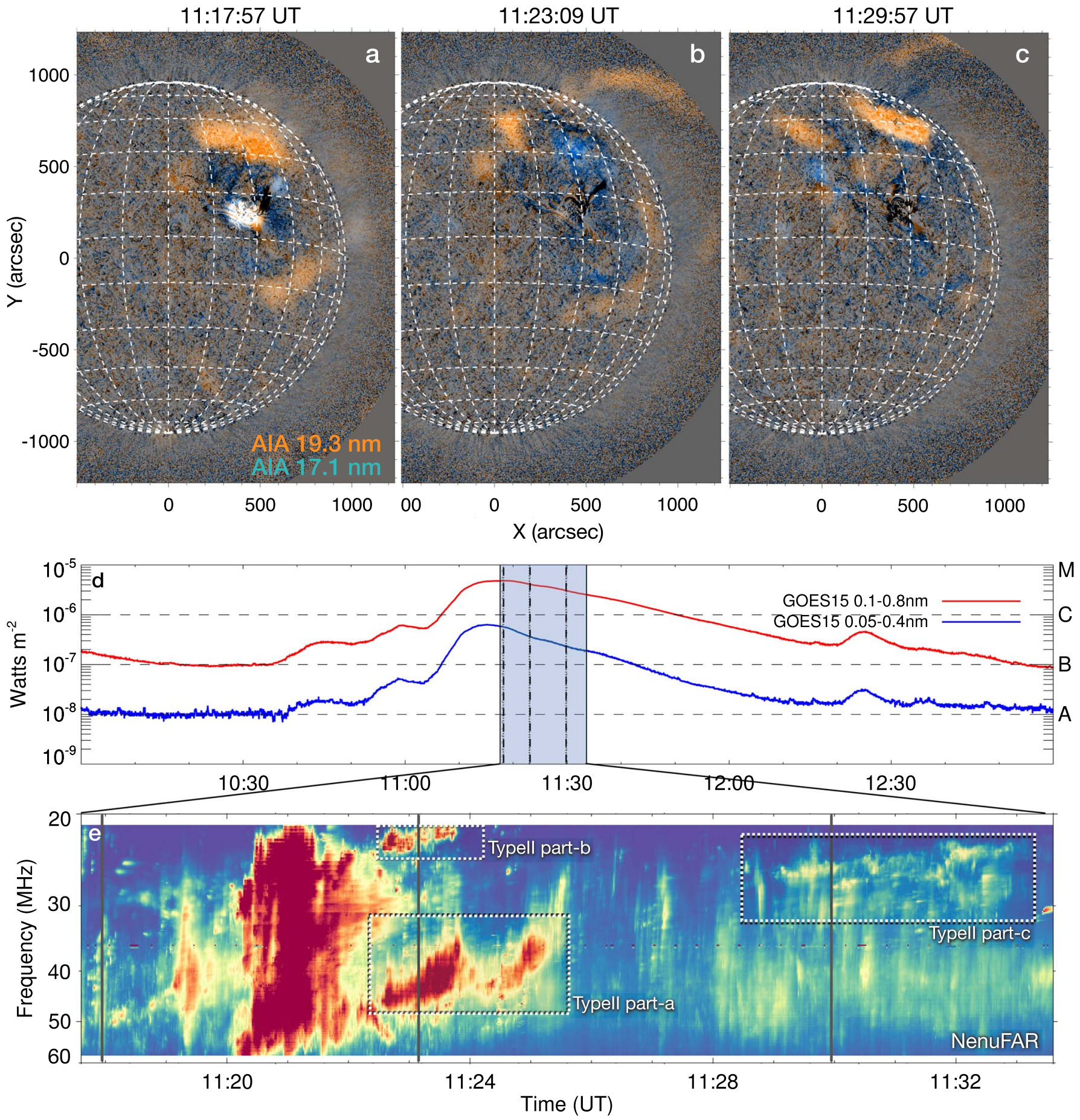

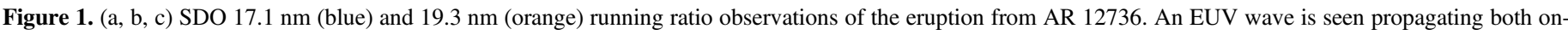

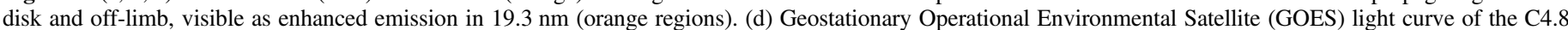

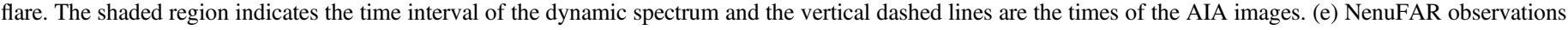

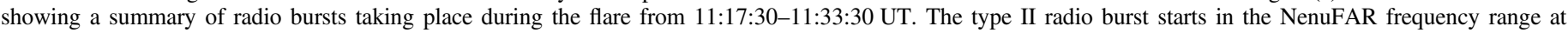

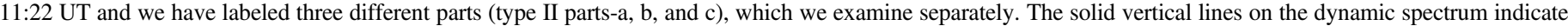
the times of the AIA images.

\subsection{Type II Parts- $a$ and -b: Harmonic and Fundamental}

Figure 2 shows our analyses of parts-a and -b of the type II burst. Type II part-a is composed of mostly of a series of fine structures known as herringbone bursts. Herringbones are relatively rare, with only $20 \%$ of type II bursts having this kind of fine structure (Cairns \& Robinson 1987). They consist of a series of forward and reverse fast-drifting bursts and are considered to be bursty electron acceleration at a coronal shock front, with electron beams propagating in opposite directions away from the shock. The type II part-a emission lane starts at $\sim 45 \mathrm{MHz}$ and drifts at a rate of $\sim-0.1 \mathrm{MHz} \mathrm{s}^{-1}$, meaning the shock responsible for the herringbones had a starting heliocentric distance of $\sim 2 R_{\odot}$ and speed of $1166 \mathrm{~km} \mathrm{~s}^{-1}$ using a Newkirk model (Newkirk 1961) - this is close to the deprojected EUV wave speed of $950 \pm 310 \mathrm{~km} \mathrm{~s}^{-1}$. 

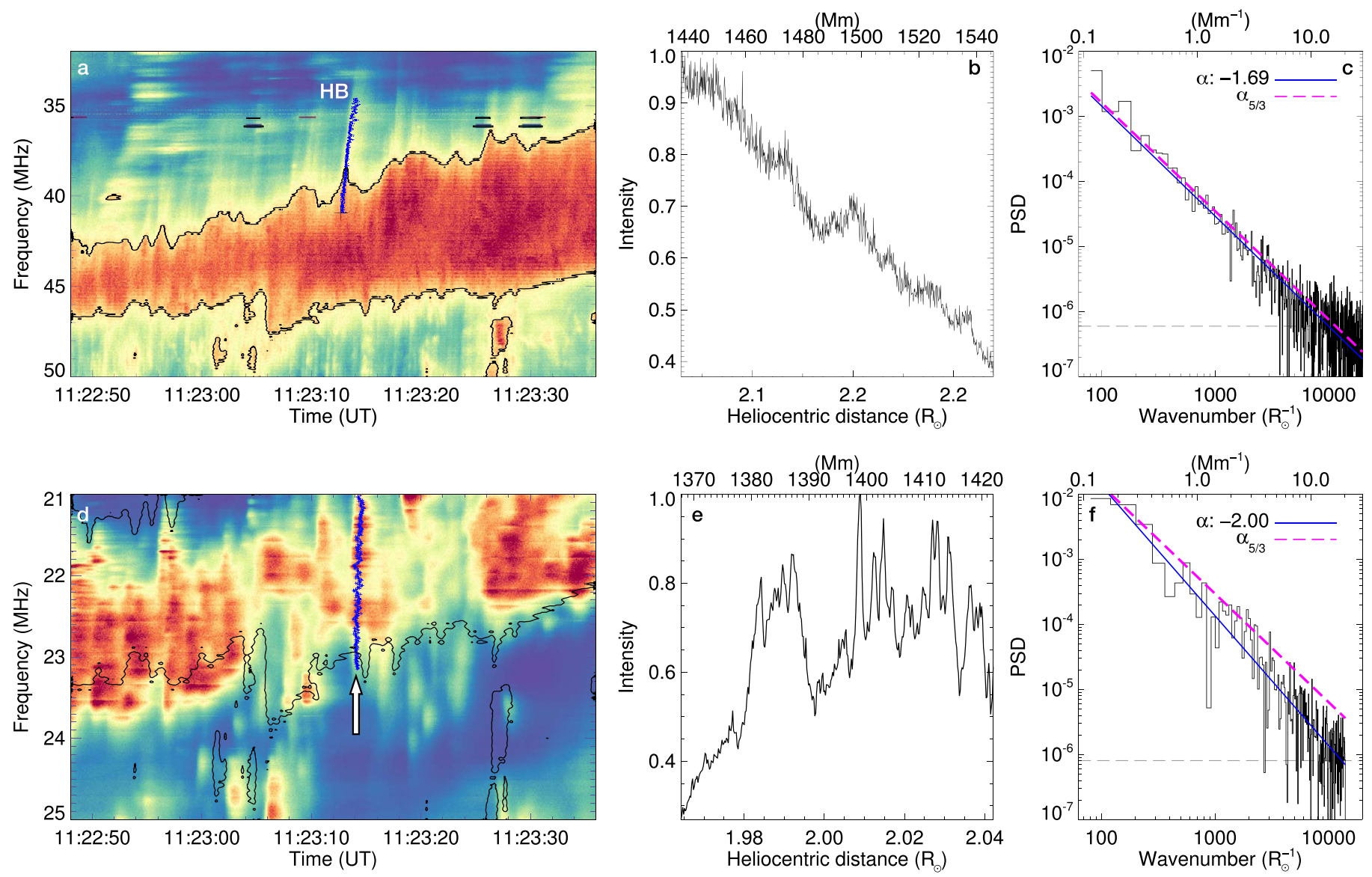

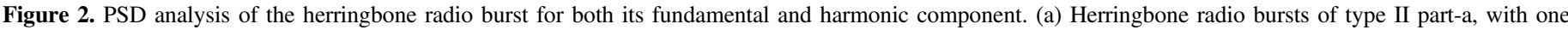

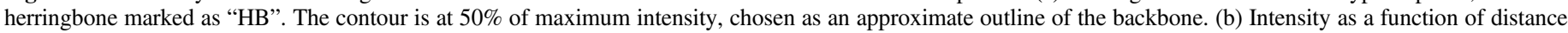

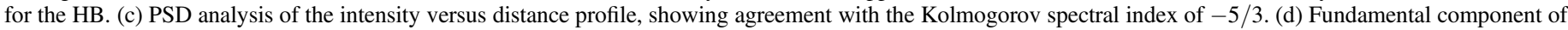

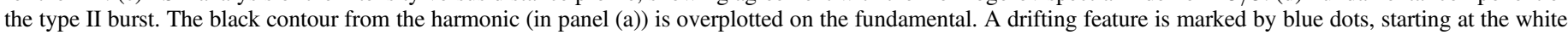
arrow. (e) Intensity versus distance for the drifting feature. (f) PSD analysis for the drifting feature, with a slightly steeper spectrum than the $-5 / 3$ value.

Type II part-b has a different appearance, beginning at approximately $23 \mathrm{MHz}$, with a more fragmented structure, see Figure 2(d). The frequency ratio of type II parts-a and -b means they are likely the fundamental $(\mathrm{F})$ and harmonic $(\mathrm{H})$ pairs of the type II burst. For example, the contour demarcating the backbone in Figure 2(a) is overplotted on Figure 2(d). This shows type II part-b is the fundamental backbone component of the radio burst. Hence, a PSD analysis on the drifting components of this F-H pair provides an opportunity to determine turbulence characteristics close to the shock surface (observed from the fundamental backbone) and the upstream region into which electron beams propagate (observed from the herringbones).

\subsection{Type II Parts- $a$ and $-b$ : PSD}

To perform the PSD, we extract an intensity versus frequency profile from a prominent herringbone radio burst occurring at $\sim 11: 23: 11 \mathrm{UT}$ and starting at $\sim 43 \mathrm{MHz}$, see blue points in Figure 2(a). As a preprocessing step, each spectrum is flattened by division of an empirical bandpass correction to account for the spectral response of NenuFAR, ensuring that any intensity enhancement is due to received flux rather than the variability in the system response across frequency. We then convert the frequency range to density using the Newkirk model, which provides us with an intensity versus distance profile. The intensity profile was resampled by interpolating to an even distance grid with $\Delta x=0.07 \mathrm{Mm}$ (distance equivalent of $\Delta f=6 \mathrm{kHz}$ at $40 \mathrm{MHz}$ ), see Figure 2(b). A PSD was performed on the intensity profile in order to obtain the distribution of coronal size scales responsible for the herringbone burst, see panel (c). A power law of the form $P(k) \sim k^{\alpha}$ is fit to the PSD, where $k$ is the wavenumber in units of inverse solar radii $\left(k=2 \pi / \lambda_{R \odot}\right)$ and $\alpha$ is the spectral index. A power-law distribution of the size scales of intensity (density) fluctuations is indicative of the scale invariance expected of a turbulent system. We find a spectral index of $\alpha=-1.69$, which matches the expectations of fully developed turbulence, e.g., with $\alpha=-5 / 3=-1.67$ (Kolmogorov 1941). We performed the same PSD analysis for 10 prominent herringbones, which resulted in an average spectral index of $\alpha_{\mu}=-1.71$. An average electron beam speed of $0.19 \mathrm{c}$ was deduced from the drift rate of the 10 herringbones (using the Newkirk model), matching previous observations (Mann \& Klassen 2005). No significant difference was found for the spectral indices or speeds between forward and reverse drift herringbones. This shows that the shock responsible for these herringbone bursts accelerated electron beams into the corona, which then propagated through a turbulent medium as they induced plasma emission.

The above result was tested with other density models that are commonly used for the metric wavelength range. For 
example, previous authors have highlighted the use of a $3.5 \times$ Saito density model (Saito et al. 1977) for type II observations at metric wavelengths (Magdalenić et al. 2010, 2012; Jebaraj et al. 2020). Such a model also produces a powerlaw distribution with an index of $\alpha=-1.72$, similar to the Newkirk model. Hence, the result is not sensitive to the choice of density model.

Continuing with the Newkirk model, we then carry out the same analysis for a drifting component of the fundamental type II part-b burst, shown in Figure 2(d) starting at 11:23:14 UT and drifting from $\sim 23-21 \mathrm{MHz}$. This drifting burst is not necessarily related to the herringbone in panel (a). It is an independent measure of emission fine structure generated in the fundamental component close to the same time as the herringbone. The power spectrum of intensity versus distance for this drifting structure again shows a power-law distribution but with a steeper index of $\alpha=-2.0$.

While the herringbone of the harmonic represents a beam propagation into the unshocked upstream corona, the fundamental backbone emission is likely a sample of the density turbulence closer to the shock surface, and its steeper PSD index may be an indicator of slightly different turbulence characteristics closer to the shock front; we discuss this further in Section 4.3.

As for the size scales of the turbulence, we find the power law exists over a range of wavenumbers from $\sim 0.1$ to $30 \mathrm{Mm}^{-1}$, similar to the wavenumbers reported in Chen et al. (2018). This means the distribution of size scales for the density inhomogeneities varies from $62 \mathrm{Mm}$ to as small as $209 \mathrm{~km}$. Values of the outer scale of density turbulence in the corona are found to be on the order of $696 \mathrm{Mm}\left(1 R_{\odot}\right)$ at a radial distance of $\sim 7 R_{\odot}$ using observations from the Ulysses and Galileo missions (Wohlmuth et al. 2001), while the inner scales of turbulent energy dissipation at $\sim 2 R_{\odot}$ are expected to be $<1$ km (Coles \& Harmon 1989; Sasikumar Raja et al. 2019). This means the size scales we derive here are in the inertial range, between the inner and outer scale of turbulence. In Section 4.4, we discuss the potential of NenuFAR to provide observation close to the inner scale (on the order of kilometers), where energy dissipation is expected to occur.

Finally, we may estimate the amplitude of the density perturbations from the intensity perturbations in the fundamental component of the radio burst (type II part-a), using the expression derived in Reid \& Kontar (2021)

$$
\frac{\Delta n}{n}=\frac{v_{\mathrm{th}}^{2}}{v_{b}^{2}} \frac{\Delta I}{I},
$$

where $\Delta n / n$ and $\Delta I / I$ are the fractional density and intensity perturbations, respectively, $v_{\text {th }}$ is the thermal speed of the plasma and $v_{b}$ is the electron beam speed. We assume a $2 \mathrm{MK}$ plasma, resulting in $v_{\text {th }}=5.5 \mathrm{Mm} \mathrm{s}^{-1}$, and the exciter speed of the drifting feature in type II part-a is found to be $78 \mathrm{Mm} \mathrm{s}^{-1}$ using a Newkirk density model. The intensity perturbations across the drifting feature produce $\Delta I / I \sim 0.14$, providing a $\Delta n / n \sim 0.8 \times 10^{-3}$, which is somewhat similar to the value of $3 \times 10^{-3}$ found from type III striae in Reid \& Kontar (2021). At larger heights than those observed here, previous radio scintillation observations have shown similar values $\Delta n / n=10^{-3}$ at $\sim 10 R_{\odot}$, with the fluctuations increasing to between $10^{-2}$ and $10^{-1}$ at distances out to $0.8 \mathrm{au}$ (Woo et al. 1995; Sasikumar Raja et al. 2016).
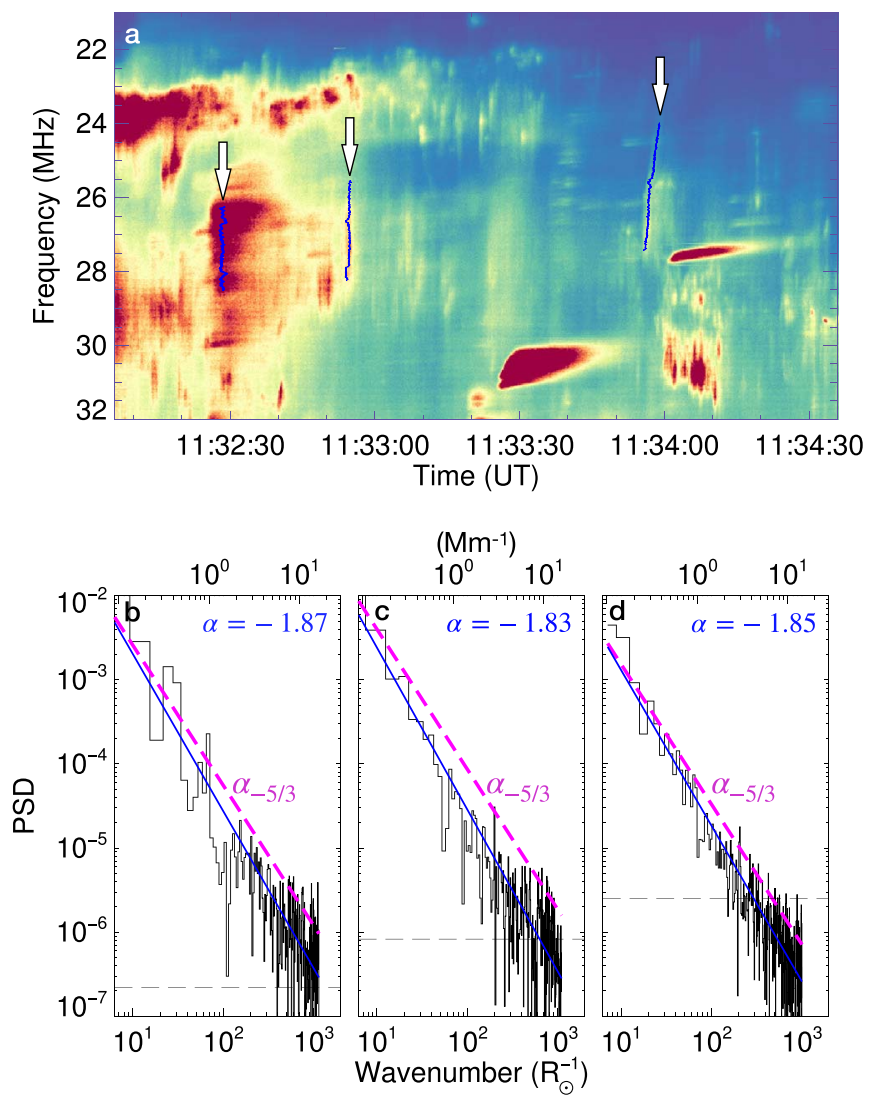

Figure 3. (a) Zoom of the region showing part-c of the type II burst (harmonic component), displaying a fine structure and fragmentation. We have identified three drifting features among this fine structure, indicated by the arrows. An intensity vs. frequency profile is extracted along these bursts (at the indicated blue points), used to perform the same PSD analysis as above. (b)-(d) A PSD for each of the drifting features in the dynamic spectrum. They again show a power-law distribution, but with slightly steeper spectral indices ( $\alpha=-1.83$ to -1.87 ) than for the harmonic herringbones.

\subsection{Type II part-c: PSD}

Part-c of the type II burst lasts from 11:28-11:38 UT starting at a frequency of $\sim 30 \mathrm{MHz}$ and drifting at a rate of $-0.022 \mathrm{MHzs}^{-1}$, see Figure 1. Given its position in the dynamic spectrum, type II part-c is a continuation of part-a of the radio burst, and we consider it to be a harmonic component. Using the Newkirk density model, the starting frequency of $15 \mathrm{MHz}$ and drift rate give a shock heliocentric distance and speed of $2.6 R_{\odot}$ and $538 \mathrm{~km} \mathrm{~s}^{-1}$, respectively. Hence, this part of the type II burst represents the shock at a larger altitude than the herringbone burst and at a slower speed.

Unlike the herringbone burst, the type II part-c burst is more fragmented and has fewer discernible drifting features, but we were able to identify three drifting bursts, see Figure 3(a). Performing the same power spectrum analysis as above shows a power-law distribution of intensity perturbations, see Figures 3(b)-(d). The power law in each is slightly steeper than the herringbone harmonic burst, showing values of $\alpha=1.83-1.87$. The steeper index may indicate that the radio emission comes from a different region in the corona with different turbulence characteristics to the region that produced the initial herringbones; this is discussed further in Section 4.3.

From Figure 1, type II part-c does not appear to be isolated but is embedded in the low frequency end of a broadband feature consisting of a number of faint forward and reverse drift bursts 
occurring at 30-55 MHz and lasting from 11:28-12:40 UT. The frequency range is indicative of an heliocentric distance of $1.5-1.8 R_{\odot}$ using a Newkirk model (assuming this is fundamental emission), and the burst frequency drifts give an exciter speeds of 0.05 c. A PSD analysis gives $\alpha=-1.64$ for the drifting bursts here, which is again a signature of turbulent plasma. This broadband feature is likely a part of the type IV burst that can also be observed in the ORFEES dynamic spectrum from 11:20-11:45 UT, extending up to frequencies of $\sim 600 \mathrm{MHz}$. The type IV burst is indicative of energetic electrons trapped in flare loops or associated with a CME (e.g., Carley et al. (2016, 2017); Morosan et al. (2019). While the type IV burst may be from plasma emission in a turbulent environment, the origin of the emission and associated electron acceleration is unclear due to the lack of images of the radio source. In future studies, the inclusion of imaging along with a PSD diagnostic of type IV fine structure could be particularly useful for analyzing the turbulent plasma properties of flare loops and CMEs.

\section{Discussion}

\subsection{Shock Location and Geometry}

The herringbone analysis provided above shows that shocks in the corona can produce bursty acceleration of electrons in a turbulent coronal environment. Unfortunately no radio imaging observations were available at the time of these radio bursts, so we cannot say where in the corona these electron beams and turbulence were located. However, type II bursts and herringbones are expected to occur in a shock with quasiperpendicular $(\sim \perp)$ geometry at the flanks of an eruption (Carley et al. 2013; Morosan et al. 2019). Given that we can image the shock propagation in EUV, we have the opportunity to determine where this disturbance may have encountered $\sim \perp$ shock geometry.

In Figures 1(a)-(b) a disturbance is visible in AIA, propagating both on-disk and and off-limb in the shape of a dome-like structure. We assume this disturbance to be a signature of the shock in EUV images. To estimate the extent of this shock, we reconstruct a spheroid in 3D space and project it onto the AIA image at the start time of the type II burst in NenuFAR ( 12:22:16 UT). The shape and position of the spheroid is adjusted by eye such that its extent across the solar surface and off-limb matches the regions of EUV emission in the AIA images, see Figure 4(a). The EUV disturbance is well described by this spheroid and allows us to roughly demarcate the 3D extent of the shock bubble. At $\sim 12: 22: 16$ UT the bubble apex was at a heliocentric distance of $\sim 2.1 R_{\odot}$.

Figure 4(b) shows this shock bubble embedded in a potential field source surface (PFSS) extrapolation of the coronal magnetic field (Stansby et al. 2020) using data from the Global Oscillation Network Group (Harvey et al. 1996). It shows that a significant amount of open field existed at the flanks of the eruption toward solar north, with the orientation of this field with respect to the shock being $\sim \perp$. While we cannot directly image the herringbone bursts, we assume the region to the north of the shock to be the most probable place for electron acceleration. Figure 4(c) shows an illustration of how a herringbone burst with a turbulent signature may be generated, e.g., with bidirectional electron beams being accelerated into turbulent plasma on open field in the upstream region of the shock.
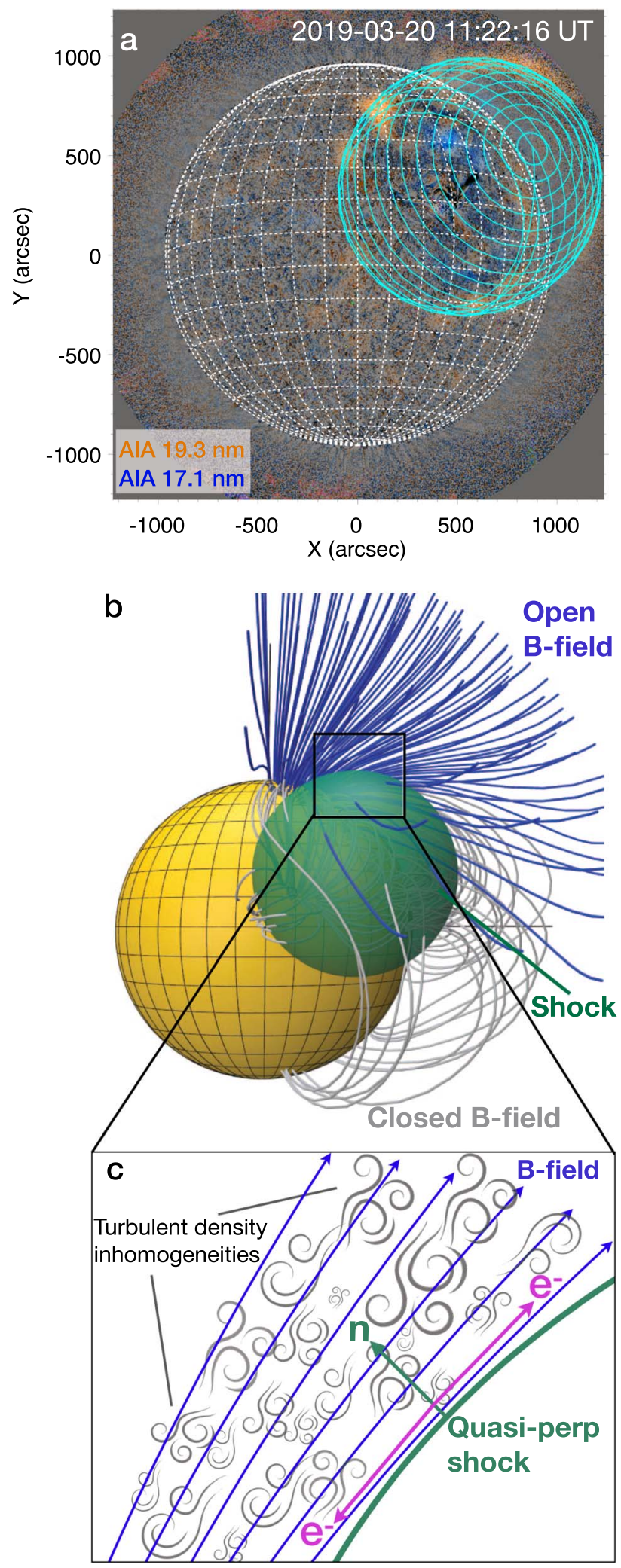

Figure 4. (a) The EUV wave propagating on-disk and off-limb is approximated by a $3 \mathrm{D}$ spheroid in the solar atmosphere, projected onto the AIA image (turquoise lines). (b) This 3D spheroid is shown as the shock surface (green sphere) embedded in a PFSS extrapolation of the magnetic field. There was a large number of open field lines to the north of the active region, where the shock normal to the magnetic field is quasi-perpendicular. (c) An illustration of the turbulent environment into which electron beams are accelerated in opposite directions. 


\subsection{Electron Beam Acceleration in Coronal Turbulence}

There are a variety of particle acceleration mechanisms that have been proposed to explain the presence of high energy particles produced by plasma shocks. One of the most common is through the so-called first order Fermi mechanism in which a charged particle undergoes repeated reflections between the upstream-downstream environment, gaining energy upon each transition (Axford et al. 1977; Drury 1983). This diffusive shock acceleration mechanism is usually employed for protons or ions, as their large gyro-radii mean they can interact with turbulent magnetic fluctuations either side of the shock to produce the repeated reflections. The particle energy gain can reach gigaelectronvolt energies (Vainio 2009), while the mechanism efficiency can depend on the shock geometry (Jokipii 1987).

For electrons the acceleration mechanism remains less clear. At low (thermal) energies electron gyro-radii are too small for interaction with magnetic perturbations in the background plasma, so they cannot experience the repeated reflections necessary for the diffusive Fermi mechanism. In order to explain how electrons are accelerated to nonthermal energies by a shock, the SDA mechanism is employed, in which the electron gains energy while undergoing a magnetic mirroring combined with a $\nabla B$-drift in the presence of the convective $E=\vec{v} \times \vec{B}$ electric field of the shock (Wu 1984; Ball \& Melrose 2001). This mechanism has been used to explain type II radio bursts as it easily produces electrons of moderate nonthermal energies (Holman \& Pesses 1983).

With the addition of turbulence in SDA, the electrons may encounter the shock multiple times and gain higher energies than just a single shock reflection (Burgess 2006). In the herringbones observed here, analysis of the electron beam energies can provide clues as to whether turbulence is involved in the acceleration mechanism. As stated above, the drift of the herringbones in Figure 2(a) provide electron beam speeds of $v_{\text {beam }}=0.19 \mathrm{c}$ (using a Newkirk model), meaning the maximum speed in the electron beam distribution is $v_{\max }=2 v_{\text {beam }}=0.38 \mathrm{c}$, or $41 \mathrm{keV}$. According to Ball \& Melrose (2001) the maximum energy gain of a particle reflected from a shock through the SDA mechanism is $E_{r} / E_{i} \leqslant 13.93$, where $E_{r}$ and $E_{i}$ are the particle reflected and incident energy, respectively. If we take the incident energy to be a few times the thermal kinetic energy of a $1 \mathrm{MK}$ plasma, then $E_{i} \sim 1 \mathrm{keV}$, meaning the electrons producing the type II part-a burst would need to experience an energy gain of up to $E_{r} / E_{i}=41$. This is beyond the single reflection limit, and would require multiple reflections of the SDA process. Several authors have modeled such multiple reflections from ripples on the shock surface in response to turbulence in the background plasma (Burgess 2006; Guo \& Giacalone 2010). In our case, the observed turbulence during herringbone production could be responsible for electron beam energy gain to $41 \mathrm{keV}$.

A statistical comparison of turbulence characteristics (amplitude and spectral index) to herringbone kinematics would help in confirming any relationship between the two phenomena. Unfortunately only 10 herringbones in part-a of the type II burst were clear enough to obtain this information, so we cannot perform such a statistical study at present. We note, however, that the PSD spectral index of the herringbone features is different to the features of type II part-c, which shows far fewer drifting features. Particular kinds of shock inhomogeneity and turbulence in the corona may be responsible for the production of herringbones. Further studies of these bursts in the context of turbulence are required, especially those that include turbulence diagnostics through imaging observations (Subramanian \& Cairns 2011).

\subsection{Evidence of Different Kinds of Turbulent Coronal Environments?}

The herringbone radio bursts show a turbulence signature of the classical Kolmogorov type, with a spectral index of $\alpha=-1.71$. However, drifting features in part-c of the type II bursts have a steeper index of $\alpha=-1.85$ and type II part-a is even steeper at $\alpha=-2.0$. What is the cause of these different spectral indices? While a steeper spectral index might be encountered beyond the turbulence inner scale, this is expected to occur at $<1 \mathrm{~km}$ at a heliocentric distance of $2 R_{\odot}$ (Sasikumar Raja et al. 2019). Given we observe size scales $>209 \mathrm{~km}$, we may rule out the presence of steep indices due to observation beyond the inner scale and discuss values of indices usually observed for inertial scales.

The turbulence spectral index for inertial scales depends on a variety of factors, including the turbulent property under investigation, e.g., whether it is velocity, magnetic field, or density perturbations. The original Kolmogorov formulation of $k^{-5 / 3}$ is predicted for velocity perturbations in an incompressible flow in a neutral medium (Kolmogorov 1941). An extension of this formulation to the compressible MHD case predicts a variety of possible spectral indices which may range from $\alpha=-1$ to -2 (Matthaeus et al. 1982; Yamauchi et al. 1998). This range of spectral indices is reflective of the various MHD wave modes a plasma may support, e.g., Alfvén waves, and fast and slow MHD waves. These wave modes lead to compressibility and anisotropy of the turbulence and can result in a departure from the incompressible, isotropic, and neutral Kolmogorov value of $k^{-5 / 3}$ (Saur et al. 2002; Shaikh \& Zank 2010; Kowal et al. 2007).

For density perturbations, which we observe here through radio burst fine structure, the story is more complex. In the nearly incompressible case dominated by magnetic fields, the density perturbations should theoretically scale similarly to pressure and follow a $k^{-7 / 3}$ (which assumes a polytropic equation of state $p \sim \rho^{\gamma}$, where $\gamma$ is the adiabatic index). However, observational work has shown that density turbulence in the corona and solar wind often follow the Kolmogorov value of $k^{-5 / 3}$ from 2-40 $R_{\odot}$ (Scott et al. 1983; Coles \& Harmon 1989; Armstrong et al. 1990). That said, MHD simulations from Kowal et al. (2007) have shown super-Alfvénic flow will produce a density spectrum of $k^{-7 / 3}$, while low Mach numbers result in $k^{-5 / 3}$. The presence of strong magnetic fields also introduces an anisotropy into the density spectra, with perturbations perpendicular to the background field following $k_{\perp}^{-2}$, while those parallel to the field follow $k_{\|}^{-5 / 3}$ (Kowal et al. 2007). Furthermore, Reid \& Kontar (2021) have recently shown that finite Langmuir wave group velocity during the plasma emission process can smooth out finer-scale variability in the emission at high spatial wavenumbers, resulting in a steepening of the spectral index in the inertial range to values less than $-5 / 3$.

It is clear that the observed spectral index for density turbulence can take on a variety of values depending on the specific conditions of the plasma environment. This may explain the different morphologies in each part of the type II burst we observe in the event reported here. For example, the herringbones are from electron beam propagation some distance away from the shock (Carley et al. 2015; Morosan et al. 2019), and likely provide a measure of density turbulence in the background corona. The drifting feature in Figure 2(d) 
crosses the fundamental emission lane, indicating the exciter may be closer to the shock surface; this region should be a more perturbed environment and larger density compressions would lead to a steeper turbulence index (Kowal et al. 2007). At the later time of the type II part-c burst the shock may have reached a different region of the corona (it occurred at a larger distance of $2.6 R_{\odot}$ ), having its own characteristic turbulence signature. This is perhaps expected, given that different regions of the solar atmosphere have been shown to have the different turbulent spectral indices ranging from $k^{-1.7}-k^{-2.3}$ (Abramenko 2005). Radio sounding experiments have also shown a steepening of density power spectra near the Kolmogorov value during the passage of a CME in the heliosphere from $<10-50 R_{\odot}$ (Woo \& Armstrong 1992; Efimov et al. 2008).

Finally, the different appearance of the type II part-c burst may also be due to the magnetic environment in which the shock is generated. For example, parts-a and -b of the radio burst were likely from a quasi-perpendicular shock geometry as described above, which produces efficient electron acceleration. On the other hand, the type II part-c burst occurred at a higher altitude where there are less quasi-perpendicular field orientations and hence less opportunities for electron acceleration; this may explain why type II part-c is a weaker section of the radio burst, similar to the analysis of Maguire et al. (2020).

\subsection{Spatial Scales of Turbulence}

As for the observed spatial scales in the inertial range of turbulence, these are limited by the spectral resolution of the instrument and the signal to noise of fine scale spectral features. We observe spatial scales from $209 \mathrm{~km}-62 \mathrm{Mm}$, in agreement with previous observations from LOFAR (Chen et al. 2018). The smallest scale that we observe is at least two orders of magnitude greater that the expected inner scale of $0.5-1 \mathrm{~km}$ over which turbulent energy dissipation occurs in the low corona (Coles \& Harmon 1989). At this scale, the spectral index of the PSD would be expected to decrease from the inertial range $(-5 / 3)$ to $<-2$, which is associated with the dissipative energy range of turbulence.

The questions is, can NenuFAR or LOFAR offer enough frequency resolution to observe the scales of turbulent energy dissipation in the corona using the technique outlined here? At $85 \mathrm{MHz}$ (the upper boundary of NenuFAR's observing range), a $6 \mathrm{kHz}$ spectral resolution results in a spatial domain resolution of $17 \mathrm{~km}$ in the corona, while the same spectral resolution at $300 \mathrm{MHz}$ results in a spatial resolution of $3.8 \mathrm{~km}$. Hence, the characteristics of instruments such as NenuFAR and LOFAR operate on the margins of being able to resolve the spectral steepening indicative of the energy dissipation associated with coronal turbulence. This of course assumes that plasma emission can be observed at such fine frequency resolution. Future studies should consider such experimentation, as observation of plasma emission and density inhomogeneity could provide insight into turbulent energy dissipation on spatial scales at which kinetic energy is thermalized and the corona is heated, see, e.g., simulations by Sokolov et al. (2013).

\section{Conclusion}

In this paper we used a PSD analysis of NenuFAR data to diagnose the distribution of spatial size scales of density turbulence in the corona that were responsible for different types of fine structure in a type II radio burst. The initial harmonic component (type II part-a) showed the most promising evidence for turbulent structure. Its herringbone fine structure showed a power-law PSD of spectral index $\alpha=-1.71$, which is close to the value of $-5 / 3$ expected for fully developed turbulence. It is likely that this turbulence existed in the coronal background, with the shock wave passing through it.

The other parts of the type II burst showed a power-law PSD signature, but with indices steeper than than the Kolmogorov value. This may be indicative of the different levels of density perturbations at the shock front or a propagation in different coronal environments.

The high time resolution, spectral resolution (and by proxy spatial resolution) that new radio telescopes such as NenuFAR and LOFAR are able to observe offer for the first time a means of determining the nature of turbulence in the corona at a range of heights and in a host of different environments, i.e., shocks, flares, and CMEs, among others. More work is needed to determine the proportion of type II radio bursts (and other types) that show this turbulent signature, allowing us to diagnose particle acceleration in the turbulent environment of coronal shocks.

E.P.C. is supported by the Schrödinger Fellowship at DIAS. K.S.R. acknowledges the financial support from the Centre National d'études Spatiales (CNES), France. This paper is based on data obtained using the NenuFAR radio telescope. NenuFAR has benefited from the following funding sources: CNRS-INSU, Observatoire de Paris-PSL, Station de Radioastronomie de Nançay, Observatoire des Sciences de l'Univers de la Région Centre, Région Centre-Val de Loire, Université d'Orléans, DIM-ACAV, and DIM-ACAV+ de la Région Ile de France, Agence Nationale de la Recherche. We acknowledge the use of the Nançay Data Center (CDN), hosted by the Nançay Radio Observatory (Observatoire de Paris-PSL, CNRS, Université d'Orléans), and also supported by Région Centre-Val de Loire. Support from Paris Astronomical Data Centre (PADC) is acknowledged for data storage and from LESIA for computing capabilities. We would like to thank the GOES and SDO teams for data access. Finally, we would like to thank the referee for their useful comments and suggestions during the review of this manuscript.

\section{ORCID iDs}

Eoin P. Carley (ํ) https://orcid.org/0000-0002-6106-5292 Baptiste Cecconi 10 https://orcid.org/0000-0001-7915-5571

Hamish A. Reid (i) https://orcid.org/0000-0002-6287-3494 Carine Briand (1) https://orcid.org/0000-0003-0271-9839 K. Sasikumar Raja (1) https://orcid.org/0000-0002-1192-1804 Sophie Masson (10) https://orcid.org/0000-0002-6376-1144 Vladimir Dorovskyy (i) https://orcid.org/0000-00034781-7061

Caterina Tiburzi 난 https://orcid.org/0000-0001-6651-4811 Nicole Vilmer (10 https://orcid.org/0000-0002-6872-3630 Pietro Zucca (1) https://orcid.org/0000-0002-6760-797X Philippe Zarka (1) https://orcid.org/0000-0003-1672-9878 Michel Tagger (1) https://orcid.org/0000-0003-2962-3220 Jean-Mathias Grießmeier (1) https://orcid.org/0000-00033362-7996

Stéphane Corbel 주 https://orcid.org/0000-0001-5538-5831 Gilles Theureau (i) https://orcid.org/0000-0002-3649-276X Alan Loh (i) https://orcid.org/0000-0002-4698-9542 Julien N. Girard (1) https://orcid.org/0000-0001-8627-0404 


\section{References}

Abramenko, V. I. 2005, ApJ, 629, 1141

Afanasiev, A. N. 2009, AnGeo, 27, 3933

Armatas, S., Bouratzis, C., Hillaris, A., et al. 2019, A\&A, 624, A76

Armstrong, J. W., Coles, W. A., Kojima, M., \& Rickett, B. J. 1990, ApJ, 358,685

Axford, W. I., Leer, E., \& Skadron, G. 1977, in 15th Int. Cosmic Ray Conf. (Trieste: PoS), 273

Bale, S. D., Badman, S. T., Bonnell, J. W., et al. 2019, Natur, 576, 237

Ball, L., \& Melrose, D. B. 2001, PASA, 18, 361

Bougeret, J. L., Kaiser, M. L., Kellogg, P. J., et al. 1995, SSRv, 71, 231

Brueckner, G. E., Howard, R. A., Koomen, M. J., et al. 1995, SoPh, 162, 357

Burgess, D. 2006, ApJ, 653, 316

Cairns, I. H., \& Robinson, R. D. 1987, SoPh, 111, 365

Carley, E. P., Long, D. M., Byrne, J. P., et al. 2013, NatPh, 9, 811

Carley, E. P., Reid, H., Vilmer, N., \& Gallagher, P. T. 2015, A\&A, 581, A100

Carley, E. P., Vilmer, N., \& Gallagher, P. T. 2016, ApJ, 833, 87

Carley, E. P., Vilmer, N., Simões, P. J. A., \& Ó Fearraigh, B. 2017, A\&A, 608, A137

Chen, X., Kontar, E. P., Yu, S., et al. 2018, ApJ, 856, 73

Chrysaphi, N., Kontar, E. P., Holman, G. D., \& Temmer, M. 2018, ApJ, 868,79

Coles, W. A., \& Harmon, J. K. 1989, ApJ, 337, 1023

Drury, L. O. 1983, RPPh, 46, 973

Efimov, A. I., Rudash, V. K., Samoznaev, L. N., et al. 2008, AdSpR, 42, 110

Guo, F., \& Giacalone, J. 2010, ApJ, 715, 406

Harvey, J. W., Hill, F., Hubbard, R. P., et al. 1996, Sci, 272, 1284

Holman, G. D., \& Pesses, M. E. 1983, ApJ, 267, 837

Jebaraj, I. C., Magdalenić, J., Podladchikova, T., et al. 2020, A\&A, 639, A56

Jokipii, J. R. 1987, ApJ, 313, 842

Kolmogorov, A. 1941, DoSSR, 30, 301

Kowal, G., Lazarian, A., \& Beresnyak, A. 2007, ApJ, 658, 423

Krupar, V., Szabo, A., Maksimovic, M., et al. 2020, ApJS, 246, 57

Lecacheux, A. 2000, Radio Astronomy at Long Wavelengths, Vol. 119 (Washington DC: American Geophysical Union), 321

Lemen, J. R., Title, A. M., Akin, D. J., et al. 2012, SoPh, 275, 17

Magdalenić, J., Marqué, C., Fallows, R. A., et al. 2020, ApJL, 897, L15

Magdalenić, J., Marqué, C., Zhukov, A. N., Vršnak, B., \& Veronig, A. 2012, ApJ, 746, 152

Magdalenić, J., Marqué, C., Zhukov, A. N., Vršnak, B., \& Žic, T. 2010, ApJ, 718,266
Maguire, C. A., Carley, E. P., McCauley, J., \& Gallagher, P. T. 2020, A\&A, 633, A56

Mann, G., \& Klassen, A. 2005, A\&A, 441, 319

Mann, G., Klassen, A., Classen, H. T., et al. 1996, A\&AS, 119, 489

Matthaeus, W. H., Goldstein, M. L., \& Smith, C. 1982, PhRvL, 48, 1256

Morosan, D. E., Kilpua, E. K. J., Carley, E. P., \& Monstein, C. 2019, A\&A, 623, A63

Nelson, G. J., \& Melrose, D. B. 1985, Type II bursts (Cambridge: Cambridge Univ. Press), 333

Newkirk, G. J. 1961, ApJ, 133, 983

Reid, H., \& Kontar, E. 2021, NatAs, 5, 796

Reid, H. A. S., \& Kontar, E. P. 2017, A\&A, 598, A44

Saito, K., Poland, A. I., \& Munro, R. H. 1977, SoPh, 55, 121

Sasikumar Raja, K., Ingale, M., Ramesh, R., et al. 2016, JGRA, 121, 605

Sasikumar Raja, K., Subramanian, P., Ingale, M., \& Ramesh, R. 2019, ApJ, 872,77

Saur, J., Politano, H., Pouquet, A., \& Matthaeus, W. H. 2002, A\&A, 386, 699

Scott, S. L., Coles, W. A., \& Bourgois, G. 1983, A\&A, 123, 207

Shaikh, D., \& Zank, G. P. 2010, MNRAS, 402, 362

Simnett, G. M., Sakai, J. I., \& Forsyth, R. J. 2005, A\&A, 440, 759

Sokolov, I. V., van der Holst, B., Oran, R., et al. 2013, ApJ, 764, 23

Stansby, D., Yeates, A., \& Badman, S. T. 2020, JOSS, 5, 2732

Subramanian, P., \& Cairns, I. 2011, JGRA, 116, A03104

Vainio, R. 2009, in IAU Symp. 257, Universal Heliophysical Processes, ed. N. Gopalswamy \& D. F. Webb (Cambridge: Cambridge Univ. Press), 413 van Haarlem, M. P., Wise, M. W., Gunst, A. W., et al. 2013, A\&A, 556, A2 Vandas, M., \& Karlický, M. 2011, A\&A, 531, A55

Vršnak, B., Aurass, H., Magdalenić, J., \& Gopalswamy, N. 2001, A\&A, 377,321

Wohlmuth, R., Plettemeier, D., Edenhofer, P., et al. 2001, SSRv, 97, 9

Woo, R., \& Armstrong, J. W. 1992, Solar Wind Seven; Proceedings of the 3rd COSPAR Colloquium (Goslar, Germany) ed. E. Marsch \& R. Schwenn, 583

Woo, R., Armstrong, J. W., Bird, M. K., \& Patzold, M. 1995, GeoRL, 22, 329

Wu, C. S. 1984, JGR, 89, 8857

Yamauchi, Y., Tokumaru, M., Kojima, M., Manoharan, P. K., \& Esser, R. 1998, JGR, 103, 6571

Zarka, P., Girard, J. N., Tagger, M., \& Denis, L. 2012, in SF2A-2012: Proc. of the Annual Meeting of the French Society of Astronomy and Astrophysics, ed. S. Boissier et al. (Paris: SF2A), 687

Zlobec, P., Messerotti, M., Karlicky, M., \& Urbarz, H. 1993, SoPh, 144, 373 